\title{
A Novel Prime Number Path Tracing Scheme Using Clos Network
}

\author{
Pankaj Singh, Gurpartap Singh \\ Department of electronics and communication, Lovely Professional University, India \\ pankaj1990@outlook.com \\ Assistant Professor, Lovely Professional University, India \\ gurpartap.15882@lpu.co.in
}

\begin{abstract}
A novel scheme of path tracing in all-optical network (AON) is described, every node has a prime number and as data packet travels in the network prime number tag is multiplied with the other node tag. At receiver end factorization is done to measure the travelled path. This technique is implemented with the help of encoder which helps to multiply the prime number tags. This scheme has highest QoS than the scheme which use optical cross connects (OXC).In this scheme OXC is replaced with the clos network which gives better performance than system with OXC.
\end{abstract}

\section{General Terms}

Network management, All-optical networks, Path tracing, Attack, WDM, OXC.

\section{Indexing terms}

Path tracing scheme, Network management, All-optical network (AON).

\section{Academic Discipline And Sub-Disciplines}

This paper belongs to Electronics and communication academic disciplines.

\section{SUBJECT CLASSIFICATION}

\author{
Optical Communication; Optical Network Management.
}

\section{COVERAGE}

This paper covers the area of the security and fault management in optical network management. It describes a novel scheme of path tracing scheme.

\section{TYPE (METHOD/APPROACH)}

Firstly previous implemented scheme was studied and implemented. A novel scheme was implemented on the basis of previous scheme it gives better results than previous schemes.

\section{INTRODUCTION}

In All-optical networks packets are always carried on a carrier that can be of different wavelengths, signal generated from one source can be delivered at the different destination nodes using wavelength division multiplexing (WDM) which helps to route the data in optical network. at each network node routing decision is made that based on its current traffic loading and the destinations addresses of the data packets received. When an optical data packet arrives at a node in network, its packet header that contains usually of lower data rate is first extracted and detected, to retrieve its destination address. The high-speed packet payload portion is buffered through fiber delay lines, before the optical packet is being switched, via the optical cross-connect (OXC), based on the routing decision formed.

For reliable data transmission and delivery in WDM networks the path should be well managed and having high monitoring facility. During the travelling time packet can be routed to wrong destination or alternate ports of the optical cross connects (OXC) due to error in switching operations and malfunctioning of switches. So at reception of the optical data packets at the destination node, identification of the exact physical network nodes or fibre links that the received optical data packets have actually traversed, is very useful to derive and estimate its complete actual physical path. This information is very useful to detect any possible network routing error due to possible malfunction of the reconfigurable optical routing devices, and diagnose the possible causes of signal quality degradation in the received optical data packets, by examining the optical impairments along the retrieved path.

Moreover, when there exists any malicious or attack traffic, it will be useful to trace down the source of the attack through examining the path information of the received packets. In addition, if path tracing is performed at certain strategic nodes in the network, the traced path information on the previously traversed network nodes or links of the data packets could be used to deduce the actual or relative amount of accumulated optical impairments or temporal delay suffered by the dynamically routed data packets. Such information is beneficial to estimate the signal quality, and make strategic scheduling and routing decisions to meet the quality of service (QoS) requirement of the network. For instance, the priority or the next-hop routing path of an optical data packet at the OXC can be re-adjusted according to its estimated previous path. Besides, possible looping of the data packets in the network can be detected and the excessively looped packets will 
be discarded. In general, it is highly desirable to have optical techniques to perform such physical path tracing on the optical layer so as to realize connection management in an optical packet-switched network in a more efficient way.

Many techniques proposed and implemented for path tracing but all techniques have some advantage and disadvantage also very first technique was pilot tone based path tracing in which pilot tones of low frequencies used to trace the path in the network. A pilot tone at a distinct frequency was added to each input port of the OXC, as the input port identifier. By examining the pilot tone frequencies contained in the switched optical signal at each output port of the OXC, the switching connections of the OXC could be derived [1], [2].After that time delay recognition technique come into picture every node have some delay instead of the pilot tones. Regarding to the time-delay recognition schemes, the optical pulses at different input or output ports in an OXC would experience different time delays, via some delay circuits. Hence, by examining the unique temporal pulse patterns generated, connection states between the input and the output ports of the OXC could be derived. The scheme could be further extended to realize path monitoring by assigning different time delay patterns to different network nodes. However, the scheme suffers from poor scalability, in terms of the amount of fibre delay required at each node in order to avoid any ambiguity among between different possible paths. Besides, precise synchronization is needed [3]-[6].

Recently, a novel of path tracing was proposed and implemented in which a distinct prime number was assigned to each node as the path information tag. Through employing optical label encoders based on prime number multiplication at the outputs of the network nodes, path information tags carried by simple computation on the optical packet label at the receiving node. Optical encoder can manipulate the labels on the fly [7].In this paper, prime number encoding scheme further extended which helps to improve the Quality of Service (Qos) of the system. Next section illustrates the principal of path tracing by employing the extended prime number encoding technique.

\section{PRIME NUMBER ENCODING SCHEME}

To trace the path in a network there are two ways one is network link tracing and other is named as network node tracing. Network link tracing identifies all the links that the optical packet has traversed. Network node tracing identifies all the nodes that the optical packet has traversed. Both of schemes can be realized using extended path tracing scheme.

\section{Network Link Tracing}

Wavelength routing network with six nodes is shown in Figure 1and Table 1. Each link is assigned with a prime number. In network link tracing every link has a prime number tag it can be 3, 7, 11, 13 etc. Whenever a signal passes through a particular link its label is multiplied with its prime number tag. Therefore, all the fibre links that the optical data packet has traversed can be identified at the receiving node, via prime-number factorization of the received label value. Figure 1 shows an example that the individual fibre links in the network have been assigned with their distinct prime-number tags. Link every link tag is multiplied with its label which is default set as 1.

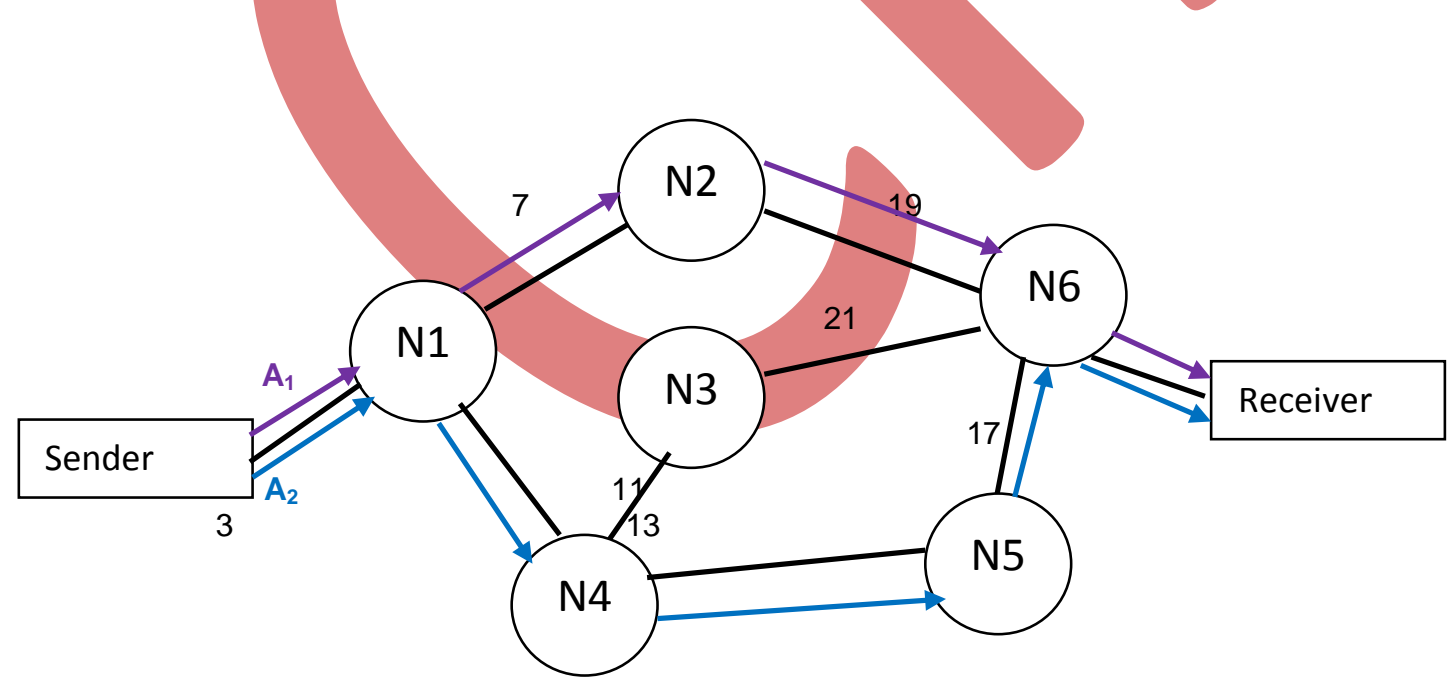

Figure 1: Example of Network link tracing.

Table 1. Example of Network link tracing.

\begin{tabular}{|c|c|c|c|c|}
\hline $\mathrm{A}_{1}$ & $\mathrm{~N} 1$ & $\mathrm{~N} 2$ & $\mathrm{~N} 6$ & \\
\hline Label Value & 1 & 7 & 133 & \\
\hline $\mathrm{A}_{2}$ & $\mathrm{~N} 1$ & $\mathrm{~N} 4$ & $\mathrm{~N} 5$ & $\mathrm{~N} 6$ \\
\hline Label value & 1 & 3 & 39 & 633 \\
\hline
\end{tabular}




\section{Network Node Tracing}

Wavelength routing network having six nodes is shown in Figure 2 and Table 2. Network node tracing is modified from network link tracing technique, in this technique each node is comprises with AWG based OXC and assigned with a prime number.

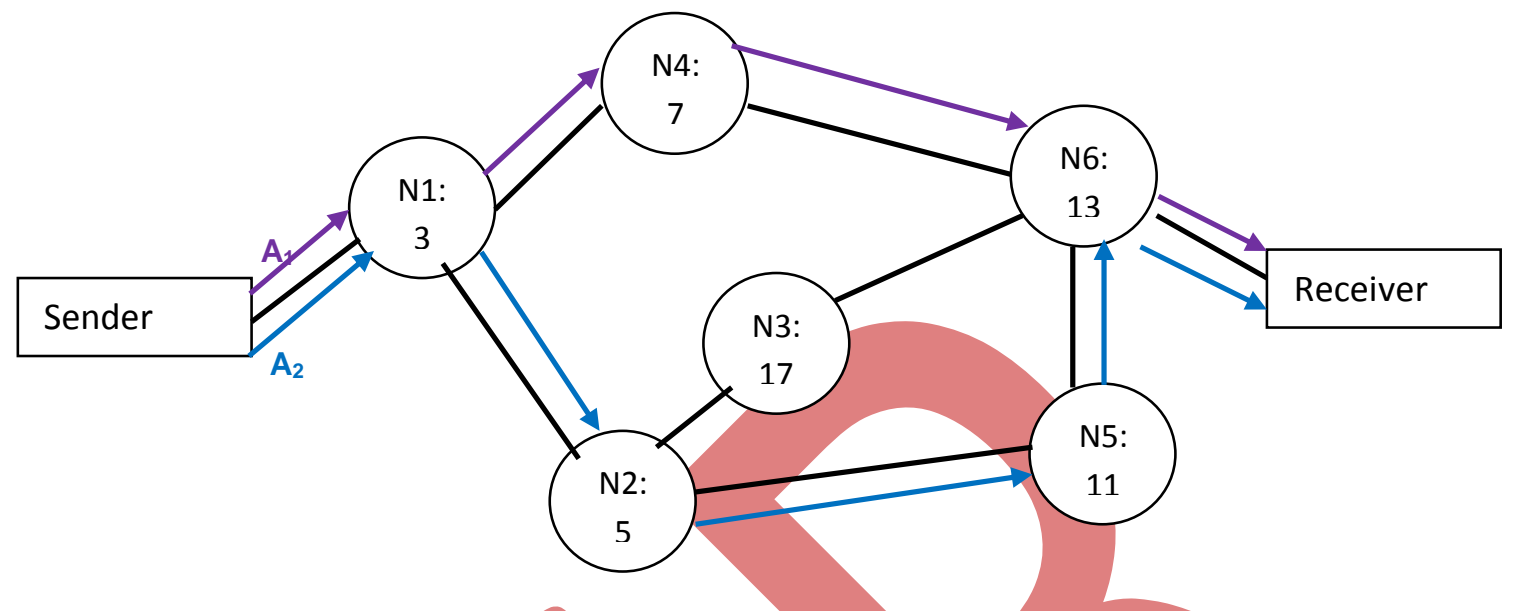

Figure 2: Example of Network node tracing.

Whenever a signal passes through a particular node its label is multiplied by the prime number of the node. Therefore, all the nodes that signal traversed identified at the receiver end, by prime number factorization of the received label value. An example of prime number encoding technique in which node assigned with a prime number is shown in Figure 2 and Table 2.

Table 2: Example of Network node tracing.

\begin{tabular}{|c|c|c|c|c|}
\hline $\mathrm{A}_{1}$ & $\mathrm{~N} 1$ & $\mathrm{~N} 2$ & $\mathrm{~N} 3$ & \\
\hline Label Value & 3 & 21 & 273 & \\
\hline $\mathrm{A}_{2}$ & $\mathrm{~N} 1$ & $\mathrm{~N} 4$ & $\mathrm{~N} 5$ & N6 \\
\hline Label value & 3 & 15 & 165 & 2145 \\
\hline
\end{tabular}

\section{EXPERIMENTAL SETUP OF PRIME NUMBER ENCODING SCHEME USING OXC}

To realize path tracing scheme laser diode is used as a source. Eight laser diodes are used to generate eight signals. These signals are divided into two parts, four signals are taken as a data signals and another four signals are taken as a pilot signals. Data signals are of 193.1, 193.2, 193.3 and $193.4 \mathrm{THz}$, all signals are having a power of 25dBm. Pilot signals frequencies are 193.5, 193.6, 193.7 and $193.8 \mathrm{THz}$, all pilot signals are having a power of $10 \mathrm{dBm}$. Further signals are modulated for transmission purpose.

Signals are further send into the optical cross connects (OXC), basically OXC is structure having MUX/DEMUX and switches. OXC helps to reduce the crosstalk in the system. After OXC signals passes through from encoder which is responsible for calculations of path tracing. Working of encoder is explained in next section.

Fully implemented experimental setup is shown in Figure 3.

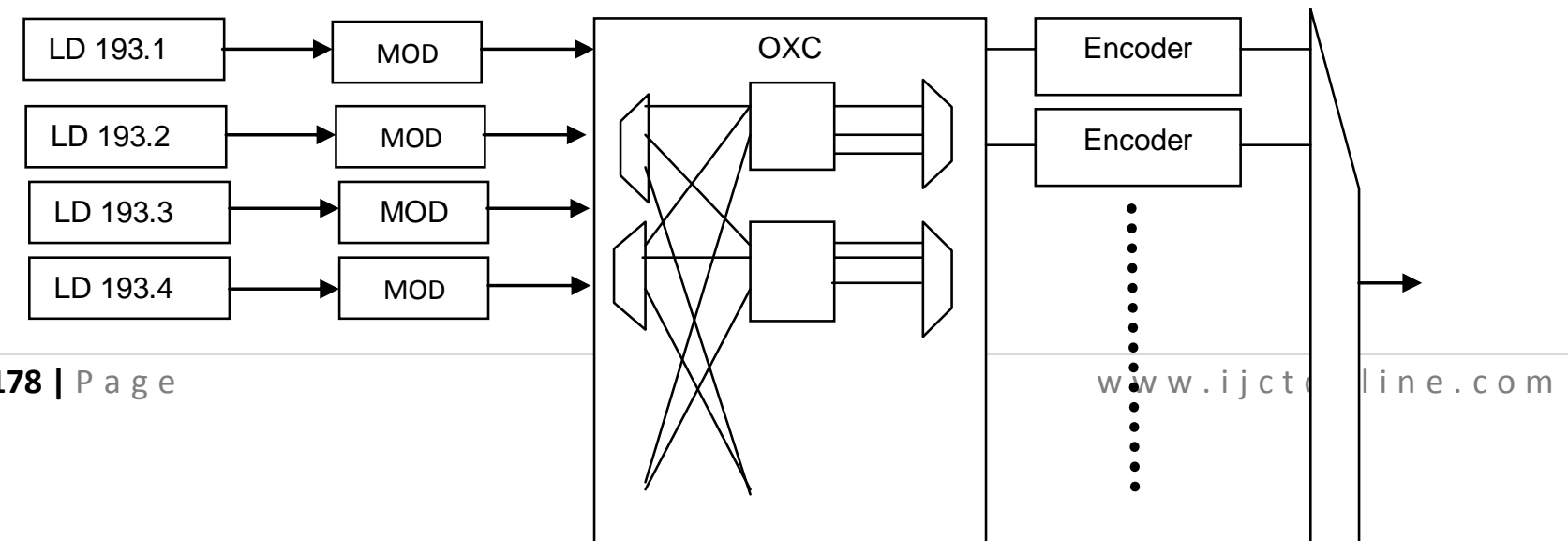




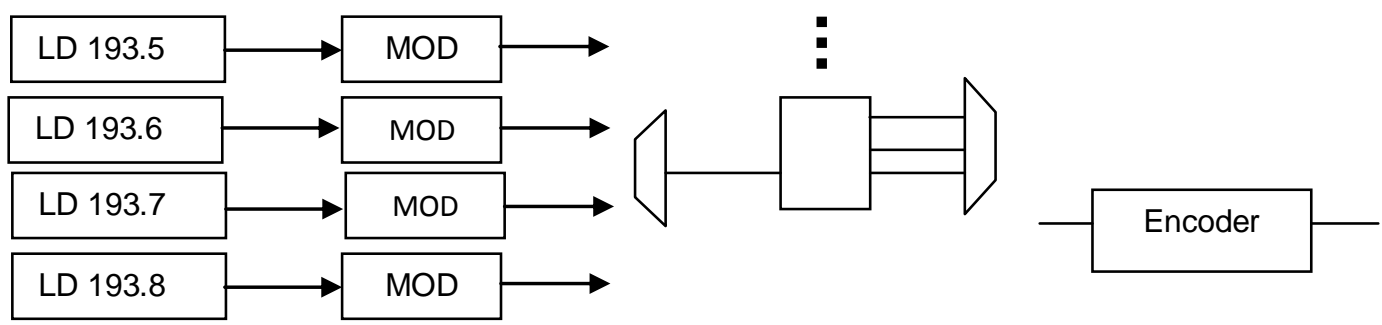

Figure 3: Experimental Setup of Prime number encoding scheme using OXC.

\section{EXPERIMENTAL SETUP OF PRIME NUMBER ENCODING SCHEME USING CLOS NETWORK}

Experimental setup of prime number encoding technique using clos network is shown in Figure 4.Eight laser diodes are used which produce eight signal having frequencies of 193.1, 193.2, 193.3, 193.4, 193.5, 193.6, 193.7 and $193.8 \mathrm{THz}$. Four signals are data signals having frequencies 193.1, 193.2, 193.3 and 193.4THz, data signals are of $25 \mathrm{dBm}$ power. Another four signals are pilot signals having frequencies of 193.5, 193.6, 193.7 and 193.8THz.All pilot signals have $10 \mathrm{dBm}$.

In this scheme OXC is replaced by clos network which is reduce the complexity and increase the power and also avoid the crosstalk like OXC. Basically clos network is a switching network it can be a multistage network for large networks. The advantage of clos network is that connection between a large number of input and output ports can be made by using only small-sized switches. A bipartite matching between the ports can be made by configuring the switches in all stages.

A multistage network is called a nonblocking if it can perform all possible connections between input and output by rearranging its connection. In such a network a connection path can always be establish between any input output pair. The clos networks can also perform all permutations in a single path without blocking. Certain subclasses can also be made non blocking if extra stages are added or connection are restricted [8]

General structure of clos network is shown in Figure 5. Clos network consist of six optical cross switches which is less from the case of OXC so complexity of the network is also reduced.

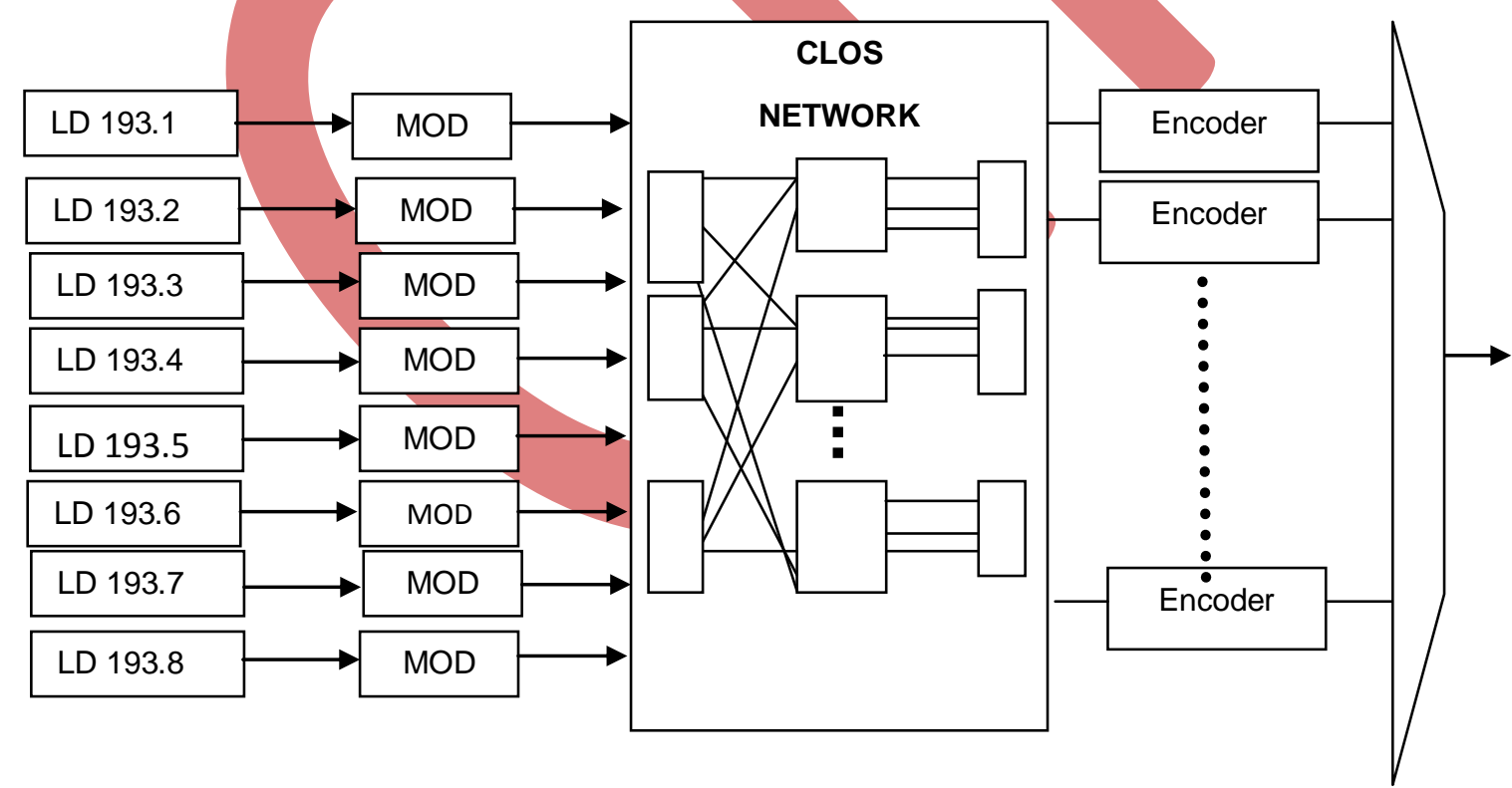

Figure 4: Experimental Setup of prime number encoding scheme using Clos network.

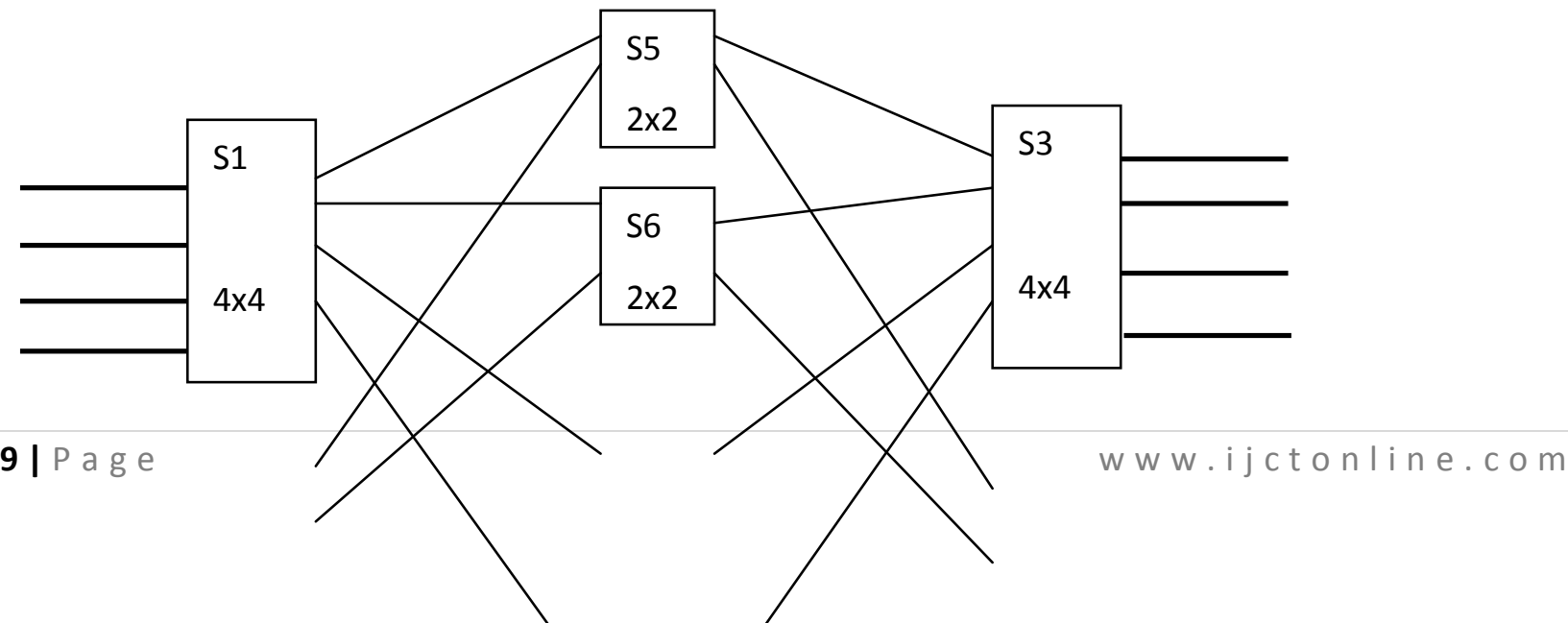




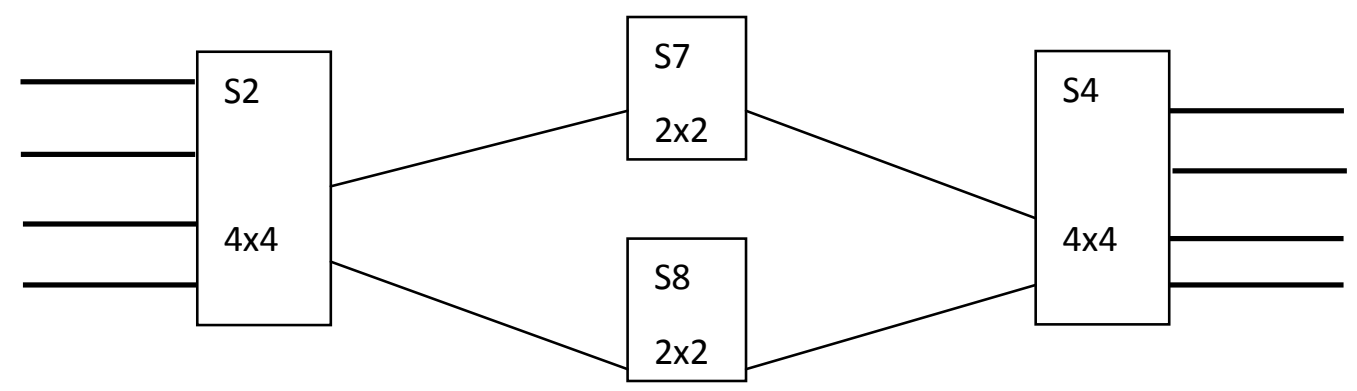

Figure 5: General diagram of Clos network.

Encoder is used as a filter, it filters the unwanted signals from data signals and pilot signals. Encoder consists of a phase shifter which gives the phase shift of 90 degree and optical delay which is required. Encoder that is a optical correlator (matching) filter is implemented using a transversal FIR architecture.

A conceptual design of an $\mathrm{N}_{\text {th }}$ order filter is shown in Figure 6 for $\mathrm{N}=8$. In this filter, the incoming optical bit pattern is divided into copies or taps. Each copy is then delayed by one bit $(\mathrm{T})$ relative to the previous one. After splitting and delaying, an Nx1 combiner is used to coherently add all delayed components. The filter response is then determined by the relative phases and amplitudes of the optical field components in the correlator arms [9].

Prime number encoding techniques are implemented and simulated on optisystem software by optiwave. As earlier discussed two types of technique are simulated, one technique is having OXC and other technique is consists of clos network. Both techniques are compared on the basis of two parameters that are Complexity and power.

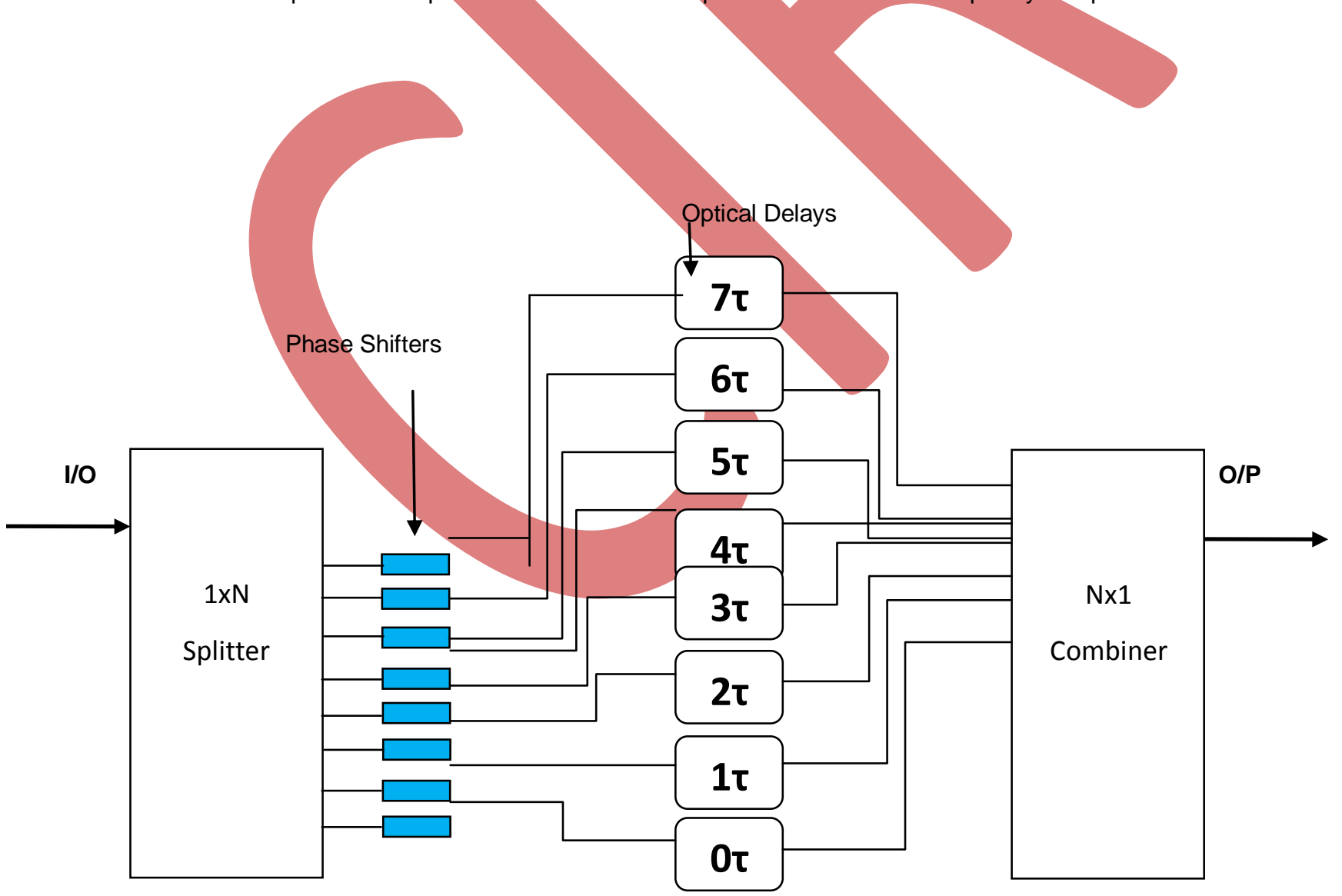

Figure 6: General diagram of optical Encoder.

Complexity is main issue in optical networking less complexity gives better results. A simple structure of optical cross connects shown in Figure 7 which is more complex than clos network, OXC consists of 24 components. These components are 8 switches and 16 MUX/DEMUXs. Therefore these components are responsible for much power consumption. MUX and DEMUXs consumes more power and they are reduced in the proposed work. MUX and DEMUXs leaks some information at every operation which leads to crosstalk. So reducing complexity also reduces the crosstalk. 
Clos network is shown in Figure 8 , which have only 8 components. It consists of only 8 switches in which 4 switches are $2 \times 2$ and remains are of $4 \times 4$. Complexity is decreased from the previous scheme; it also helps to increase the power of the system.

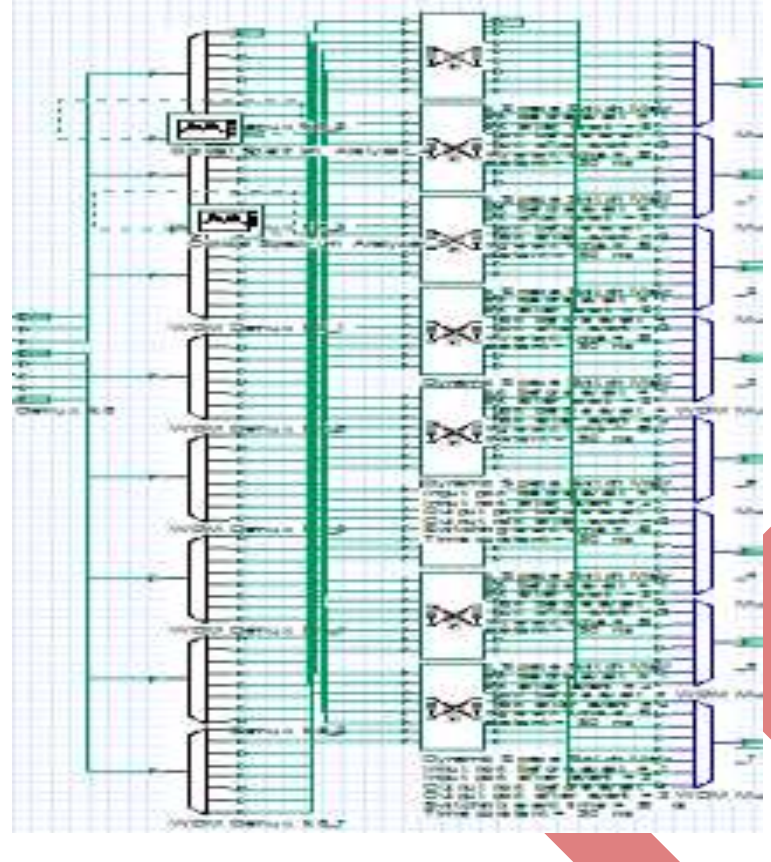

Figure 7: Implemented optical cross connects (OXC).

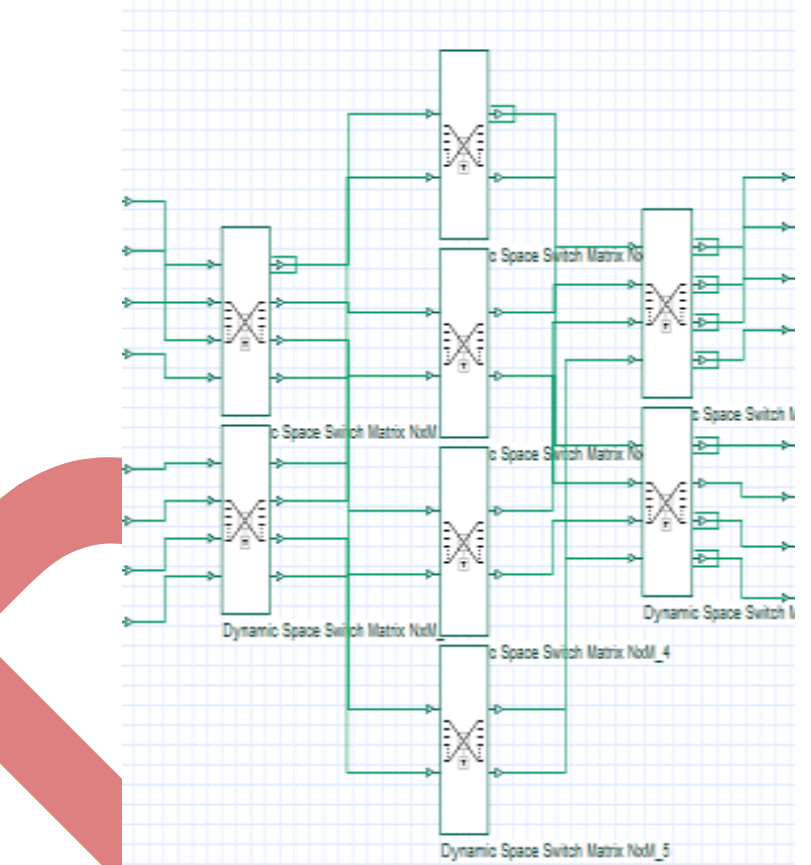

Figure 8: Implemented clos network.
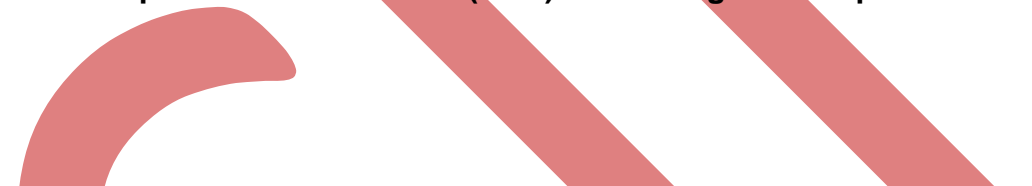

Power is also a important factor in optical communication. Rower received at receiver side matters a lot, signal having high power will travels more distance. Power received in prime number encoding scheme using OXC is only $7.269 \mathrm{dBm}$ as shown in Figure 9. Optical spectrum analyzer of this technique is shown in Figure 10; it shows the power at individual frequency. Data signals have increased power than pilot signals and also without crosstalk.

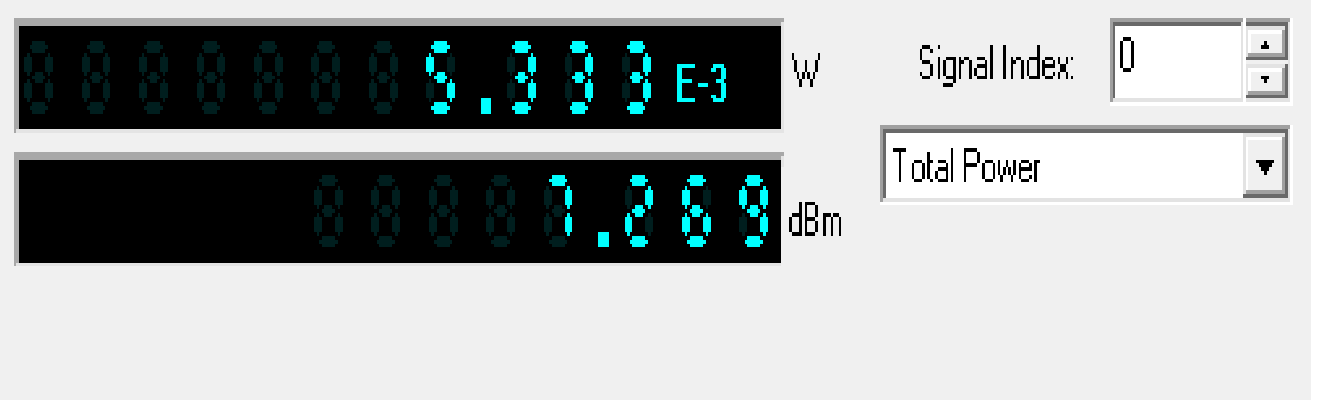

Figure 9: Power meter showing total received power of prime number encoding technique using OXC. 


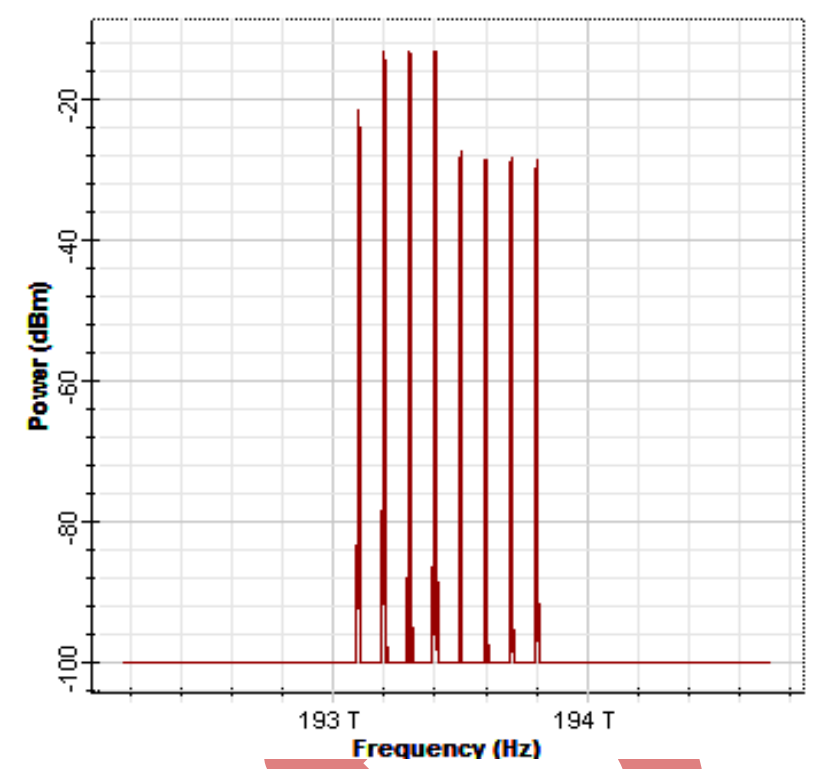

Figure 10: Optical spectrum of received signal in prime number encoding technique using OXC.

Total power received for the prime number encoding technique using clos network is shown in Figure 11 which is much higher than the value of power as compared to the prime number encoding technique using OXC, it is almost double. Clos network technique having the power of $15.339 \mathrm{dBm}$.

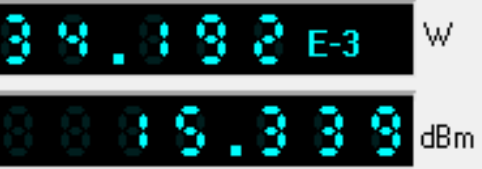

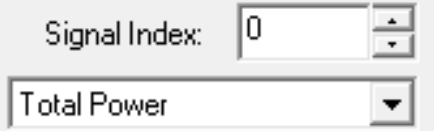

Figure 11: Total received power for prime number encoding technique using clos network.

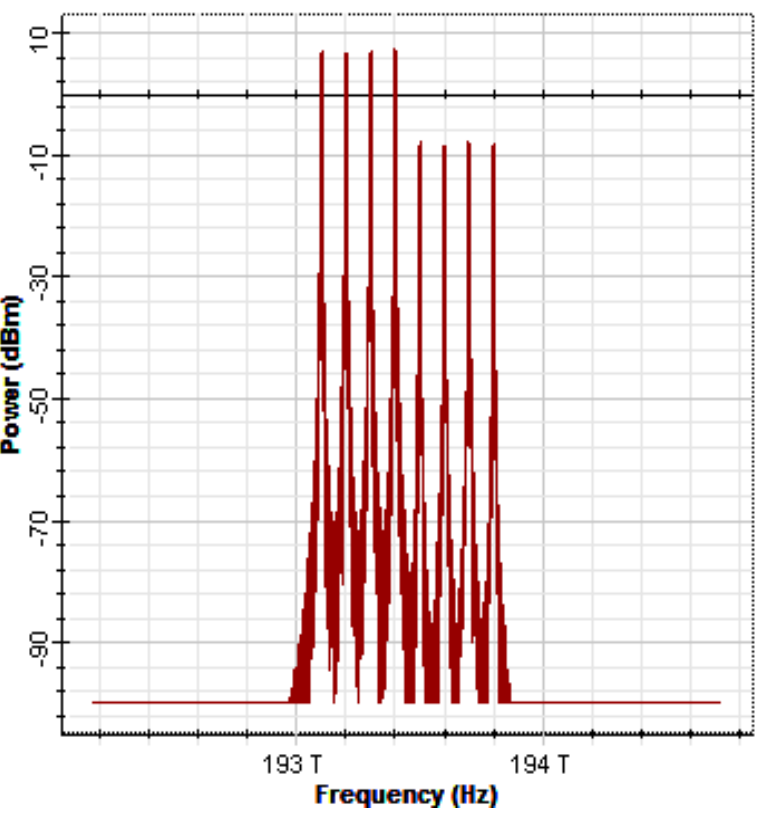

Figure 12: Optical Spectrum of prime number encoding technique using clos network.

Optical spectrum of received signals is shown in Figure 12. It shows the power of individual frequencies, power of data signals that are first four signals are higher than the power of pilot signals that are remaining four signals. Therefore from 
the above results it is clear that by replacing OXC with clos network gives better performance in terms of power and it also reduces the complexity.

\section{CONCLUSION}

Complexity and power are two important factors in optical communication. To measure and improve the both factors two techniques are implemented, first is prime number encoding technique using OXC and second is prime number encoding technique using clos network. Technique using clos network gives better performance in terms of power and it also reduces the complexity. So clos network can be used in the place of OXC to obtain better results.

\section{ACKNOLEDGEMENT}

First of all I would like to thank Gurpartap Singh for his support to make this research paper complete and I would like to thank to my parents for their all kind of help, without their help this work cannot be completed. Thanks again to everyone who so ever supported me.

\section{REFERENCES}

[1] E.Kong, F.Tong, K.P.Ho, L.K.Chen, \& C.K.Chan.1999."Pilot-tone based optical path supervisory scheme for optical cross-connects," Electronics Letter, vol. 35, no. 17, pp. 1481-1483.

[2] S. Zhong, W. Chen, X.-H. Yang \& Y.-J. Chen.2000. "Transparent optical path and crosstalk monitoring scheme for arrayed waveguide grating-based optical cross connect," IEEE Photon. Technology Letters, vol. 12, no.9, pp. 1249-1251.

[3] C.C.Lee,T.C.Kao,H.C.Chien,\& S.Chi (2005."A novel supervisory scheme for OXC based on different time-delay recognition," IEEE Photonics Technology Letters, vol. 17, no. 12, pp. 2745-2747.

[4] J. Yu, L. K. Chen, G.-W. Lu \& S.-T. Ho.2006. "An improved OXC supervisory scheme based on different time delay recognition," in Proc. Joint Int. Conf. Optical Internet and Next Generation Network (COIN-NGNCON), Jeju, Korea, Paper PS-13.

[5] Z.Qian, G.W.Lu \& S.-Y.Li.2006."Efficient OXC monitoring based on time delay recognition," in Proc. Int. Conf. Transparent Optical Networks (ICTON), Nottingham, U.K., Paper We.B3.4.

[6] Z.Qian,G.W.Lu,andS.-Y.Li.2008."Improved optical path supervisory scheme for optical cross connects based on different time-delay recognition," OSA J. Optical Networks, vol. 7, no. 1, pp. 80-87.

[7] K. H. Tse \& C. KitChan.2012."A Path Tracing Scheme for All-Optical Packet-Switched Networks", IEEE Journal Of Lightwave Technology, Vol. 30, Issue. 11,pp. 1625-1631.

[8] Kai Hwang.2001. Advance Computer Architecture, Tata McGraw-Hill Publications.

[9] M. S. Rasras.2008."A programmable 8-bit optical correlator filter for optical bit pattern recognition," IEEE Photon. Technology Letters, vol. 20, no. 9, pp. 694-696.
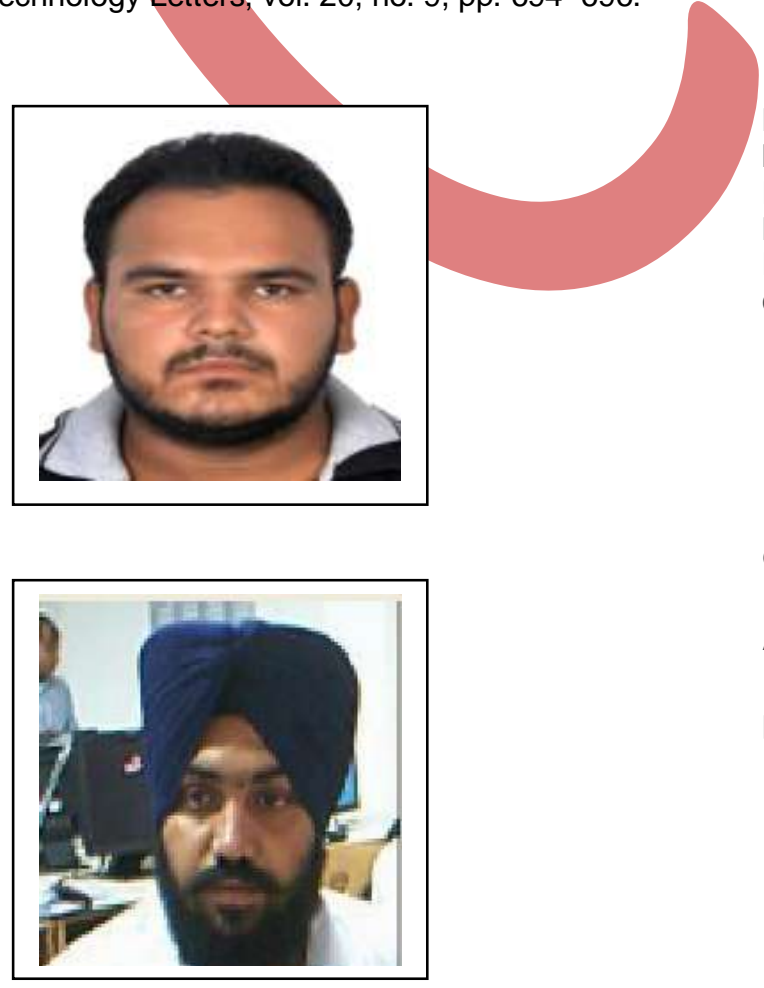

He was born in Sri Ganganagar, Rajasthan (INDIA). He has done his B.Tech (Hons.)- ECE from Lovely Professional University and currently he is pursuing his M.Tech- ECE from Lovely Professional University, Phagwara (INDIA). His research area is optical communication.

He was born in Tarn Taran, Punjab (INDIA). He has done his B.Tech \& M.Tech from Lovely Professional University, Phagwara (INDIA). Now he is serving as Assistant Professor in Lovely Professional University, Phagwara (INDIA). He has published a number of papers in various international journals. His main area of research is Wireless Optics and Optical communication. 